TAMKANG JOURNAL OF MATHEMATICS

Volume 33, Number 2, Summer 2002

\title{
A SOLUTION OF ONE PROBLEM OF COMPLEX INTEGRATION
}

\author{
ŽIVORAD TOMOVSKI AND KOSTADIN TRENČEVSKI
}

Abstract. In this paper the following identity

$$
\left(\sqrt{\pi}+\int_{0}^{i} e^{-1 / t^{2}} d t\right) e^{-1}=i\left(1-\frac{2^{1}}{1}+\frac{2^{2}}{1 \cdot 3}-\frac{2^{3}}{1 \cdot 3 \cdot 5} \cdots\right)
$$

is proved, where the integration is done over a curve with tangent vector at 0 toward the positive part of $x$-axis.

\section{Formulation of the problem.}

K. Trenčevski has set the following numerical expansion

$$
\left(\sqrt{\pi}+\int_{0}^{i} e^{-1 / t^{2}} d t\right) e^{-1}=i\left(1-\frac{2^{1}}{1}+\frac{2^{2}}{1 \cdot 3}-\frac{2^{3}}{1 \cdot 3 \cdot 5} \ldots\right)
$$

where the integration is done over a curve with tangent vector at 0 toward the positive part of $x$-axis.

This problem has not appeared until now in the literature and it is a subject of our consideration. However, applying some known results obtained by D. S. Mitrinović, J. D. Kečkić [1] and M. R. Spiegel [2], this identity finally is proved by the author Ž. Tomovski.

\section{Solution of the problem.}

Let $F(z)=\int_{z}^{\infty} \frac{e^{-t}}{t^{p}} d t$, where $p>0$ and Rez $>0$. In [2], p.288-289, was proved the following identity

$$
\begin{aligned}
F(z)= & e^{-z}\left[\frac{1}{z^{p}}-\frac{p}{z^{p+1}}+\frac{p(p+1)}{z^{p+2}}-\ldots+(-1)^{n} \frac{p(p+1)(p+2) \cdots(p+n-1)}{z^{p+n}}\right] \\
& +(-1)^{n+1} p(p+1)(p+2) \cdots(p+n) \int_{z}^{\infty} \frac{e^{-t}}{t^{p+n+1}} d t
\end{aligned}
$$

Received April 11, 2001; revised September 10, 2001.

2000 Mathematics Subject Classification. 30B50, 65D30.

Key words and phrases. Numerical expansion, Poisson integral, identity. 
We have

$$
A=\left(\sqrt{\pi}+\int_{0}^{i} e^{-1 / t^{2}} d t\right) e^{-1}=\left(\sqrt{\pi}+\int_{0}^{1} e^{-1 / t^{2}} d t+\int_{1}^{i} e^{-1 / t^{2}} d t\right) e^{-1}
$$

Let us evaluate the integral $I=\int_{0}^{1} e^{-1 / t^{2}} d t$. By putting $u=1 / t$ and applying the identity (2.1), we obtain

$$
\begin{aligned}
I= & \frac{1}{2}\left[e^{-1}\left(1-\frac{3}{2}+\frac{3 \cdot 5}{2^{2}}-\ldots+(-1)^{n} \frac{1 \cdot 3 \cdot 5 \cdots(2 n+1)}{2^{n}}\right)\right. \\
& \left.+(-1)^{n+1} \frac{1 \cdot 3 \cdot 5 \cdots(2 n+3)}{2^{n+1}} \int_{1}^{\infty} \frac{e^{-u}}{u^{n+5 / 2}} d u\right]
\end{aligned}
$$

On the other hand,

$$
\sqrt{\pi}=2 \int_{0}^{\infty} e^{-u^{2}} d u=2 \int_{0}^{1} e^{-u^{2}} d u+2 \int_{1}^{\infty} e^{-u^{2}} d u
$$

It is obvious that $\int_{0}^{1} e^{-t^{2}} d t=\sum_{n=0}^{\infty} \frac{(-1)^{n}}{n !} \frac{1}{2 n+1}$. From (2.1), we get

$$
\begin{aligned}
\int_{1}^{\infty} e^{-u^{2}} d u= & \frac{1}{2} \int_{1}^{\infty} e^{-t} t^{-1 / 2} d t \\
= & \frac{1}{2}\left[e^{-1}\left(1-\frac{1}{2}+\frac{1 \cdot 3}{2^{2}}-\ldots+(-1)^{n} \frac{1 \cdot 3 \cdot 5 \cdots(2 n-1)}{2^{n}}\right)\right. \\
& \left.+(-1)^{n+1} \frac{1 \cdot 3 \cdot 5 \cdots(2 n+1)}{2^{n+1}} \int_{1}^{\infty} \frac{e^{-u}}{u^{n+3 / 2}} d u\right]
\end{aligned}
$$

Integrating by parts, we obtain

$$
\int_{1}^{\infty} \frac{e^{-u}}{u^{n+3 / 2}} d u=e^{-1}-\frac{2 n+3}{2} \int_{1}^{\infty} \frac{e^{-u}}{u^{n+5 / 2}} d u .
$$

Thus,

$$
\begin{aligned}
\int_{1}^{\infty} e^{-u^{2}} d u= & \frac{1}{2}\left[e^{-1}\left(1-\frac{1}{2}+\frac{1 \cdot 3}{2^{2}}-\ldots+(-1)^{n+1} \frac{1 \cdot 3 \cdot 5 \cdots(2 n+1)}{2^{n+1}}\right)\right. \\
& \left.+(-1)^{n+2} \frac{1 \cdot 3 \cdot 5 \cdots(2 n+3)}{2^{n+2}} \int_{1}^{\infty} \frac{e^{-u}}{u^{n+5 / 2}} d u\right]
\end{aligned}
$$

Hence,

$$
\begin{aligned}
\sqrt{\pi}= & 2 \sum_{s=0}^{\infty} \frac{(-1)^{s}}{s !} \frac{1}{2 s+1}+e^{-1}\left(1-\frac{1}{2}+\frac{1 \cdot 3}{2^{2}}-\ldots+(-1)^{n+1} \frac{1 \cdot 3 \cdot 5 \cdots(2 n+1)}{2^{n+1}}\right) \\
& +(-1)^{n+2} \frac{1 \cdot 3 \cdot 5 \cdots(2 n+3)}{2^{n+2}} \int_{1}^{\infty} \frac{e^{-u}}{u^{n+5 / 2}} d u
\end{aligned}
$$


According to (2.2) and (2.3), we obtain

$$
\sqrt{\pi}+\int_{0}^{1} e^{-1 / t^{2}}=2 \sum_{s=0}^{\infty} \frac{(-1)^{s}}{s !} \frac{1}{2 s+1}+e^{-1} .
$$

But,

$$
\begin{aligned}
\int_{1}^{i} e^{-1 / t^{2}} d t & =\int_{1}^{i}\left(\sum_{s=0}^{\infty} \frac{1}{s !}(-1)^{s} \frac{1}{t^{2 s}}\right) d t \\
& =\sum_{s=0}^{\infty} \frac{(-1)^{s}}{s !(2 s-1)}-i \sum_{s=0}^{\infty} \frac{1}{s !(2 s-1)}
\end{aligned}
$$

Then, according to (2.4) and (2.5), we obtain

$$
\begin{aligned}
A & =\left[2 \sum_{s=0}^{\infty} \frac{(-1)^{s}}{s !} \frac{1}{2 s+1}+\sum_{s=0}^{\infty} \frac{(-1)^{s}}{s !(2 s-1)}+e^{-1}-i \sum_{s=0}^{\infty} \frac{1}{s !(2 s-1)}\right] e^{-1} \\
& =\left[2 \sum_{s=0}^{\infty} \frac{(-1)^{s}}{s !} \frac{1}{2 s+1}-1+\sum_{s=0}^{\infty} \frac{(-1)^{s+1}}{(s+1) !(2 s+1)}+e^{-1}-i \sum_{s=0}^{\infty} \frac{1}{s !(2 s-1)}\right] e^{-1} \\
& =\left[\sum_{s=0}^{\infty} \frac{(-1)^{s}}{s !(2 s+1)}\left(2-\frac{1}{s+1}\right)-1+e^{-1}-i \sum_{s=0}^{\infty} \frac{1}{s !(2 s-1)}\right] e^{-1} \\
& =\left[\sum_{s=0}^{\infty} \frac{(-1)^{s}}{(s+1) !}-1+e^{-1}-i \sum_{s=0}^{\infty} \frac{1}{s !(2 s-1)}\right] e^{-1} \\
& =-i \sum_{s=0}^{\infty} \frac{1}{s !(2 s-1)} \sum_{s=0}^{\infty} \frac{(-1)^{s}}{s !}
\end{aligned}
$$

Let $a_{s}=\frac{(-1)^{s}}{s !}, b_{s}=\frac{1}{s !(2 s-1)}$. Then,

$$
\left(\sum_{s=0}^{\infty} a_{s}\right)\left(\sum_{s=0}^{\infty} b_{s}\right)=\sum_{s=0}^{\infty} c_{s}
$$

where

$$
\begin{aligned}
c_{s} & =\sum_{k=0}^{s} a_{s-k} b_{k}=\sum_{k=0}^{s} \frac{(-1)^{s-k}}{(s-k) !} \cdot \frac{1}{k !(2 k-1)} \\
& =\frac{(-1)^{s}}{s !} \sum_{k=0}^{s} \frac{(-1)^{k}}{2 k-1} \frac{s !}{k !(s-k) !} \\
& =\frac{(-1)^{s}}{s !} \sum_{k=0}^{s}(-1)^{k}\left(\begin{array}{l}
s \\
k
\end{array}\right) \frac{1}{2 k-1} .
\end{aligned}
$$


Then we shall apply the following identity, obtained by D. S. Mitrinović and J. D. Kečkić in [1], p.146.

$$
\sum_{k=0}^{n}(-1)^{k}\left(\begin{array}{l}
n \\
k
\end{array}\right) \frac{1}{a k+b}=\frac{n ! a^{n}}{b(a+b)(2 a+b) \cdots(n a+b)} .
$$

The left-hand side of this identity is in fact, equal to $(1 / b) F(-n, b / a ; 1+b / a ; 1)$ and so it is merely a specialized version of the Chu-Vandermonde summation theorem for the finite Gauss hypergeometric series: $F(-n, b ; c ; 1)$ with $b$ replaced by $b / a$ and $c=1+b / a$. If $a=2$ and $b=-1$, we obtain

$$
\sum_{k=0}^{s}(-1)^{k}\left(\begin{array}{l}
s \\
k
\end{array}\right) \frac{1}{2 k-1}=\frac{s ! 2^{s}}{(-1) \cdot(2-1) \cdot(2 \cdot 2-1) \cdots(2 s-1)}=-\frac{s ! 2^{s}}{(2 s-1) ! !}
$$

i.e.

$$
c_{s}=(-1)^{s+1} \frac{2^{s}}{(2 s-1) ! !}
$$
that

Finally, $A=i \sum_{s=0}^{\infty}(-1)^{s} \frac{2^{s}}{(2 s-1) ! !}$, where $\left.(2 s-1) ! !\right|_{s=0}=1$. In the last step we used

$$
(2 s-1) ! !=1 \cdot 3 \cdot 5 \cdots(2 s-1)=\frac{1 \cdot 2 \cdot 3 \cdot 4 \cdots(2 s-1)(2 s)}{2 \cdot 4 \cdot 6 \cdots(2 s)}=\frac{(2 s) !}{2^{s} s !}
$$

and hence

$$
\left.(2 s-1) ! !\right|_{s=0}=\left.\frac{(2 s) !}{2^{s} s !}\right|_{s=0}=\frac{0 !}{2^{0} 0 !}=1 .
$$

That finishes the proof.

Remark. In [3] was proved the following numerical identity

$$
1+\sum_{s=1}^{\infty} \frac{2^{s}}{(2 s-1) ! !}=e\left(\sqrt{\pi}+\int_{0}^{1} e^{-1 / t^{2}} d t\right)
$$

Applying this identity, the proof of our problem can be refined. Indeed,

$$
\begin{aligned}
A & =\left(\sqrt{\pi}+\int_{0}^{1} e^{-1 / t^{2}} d t\right) e^{-1}+e^{-1} \int_{1}^{i} e^{-1 / t^{2}} d t \\
& =e^{-1}\left[\sum_{s=0}^{\infty} \frac{(-1)^{s}}{s !} \sum_{s=0}^{\infty} \frac{2^{s}}{(2 s-1) ! !}+\sum_{s=0}^{\infty} \frac{(-1)^{s}}{s !(2 s-1)}-i \sum_{s=0}^{\infty} \frac{1}{s !(2 s-1)}\right]
\end{aligned}
$$

But,

$$
\begin{aligned}
\sum_{s=0}^{\infty} \frac{(-1)^{s}}{s !} \sum_{s=0}^{\infty} \frac{2^{s}}{(2 s-1) ! !} & =\sum_{s=0}^{\infty}\left(\sum_{k=0}^{s} \frac{(-1)^{s-k}}{(s-k) !} \frac{2^{k}}{(2 k-1) ! !}\right) \\
& =\sum_{s=0}^{\infty} \frac{(-1)^{s}}{s !} \sum_{k=0}^{s}(-1)^{k} \frac{s !}{(s-k) ! k !} \frac{k ! 2^{k}}{(2 k-1) ! !} \\
& =\sum_{s=0}^{\infty} \frac{(-1)^{s}}{s !} \sum_{k=0}^{s}(-1)^{k}\left(\begin{array}{l}
s \\
k
\end{array}\right) \frac{(2 k) ! !}{(2 k-1) ! !}
\end{aligned}
$$


Then, we shall apply the well-known formulae (see [1], p145)

$$
\sum_{k=0}^{n}(-1)^{k}\left(\begin{array}{l}
n \\
k
\end{array}\right) f(k)=(-1)^{n} \Delta^{n} f(0)
$$

where $f(k)=\frac{(2 k) ! !}{(2 k-1) ! !}$ and $\Delta^{n} f$ is the finite difference of $f$ of order $n$. Applying the mathematical induction of $n$, we get

$$
\Delta^{n} f(k)=(-1)^{n-1} \frac{(2 k) ! !}{(2 n+2 k-1) ! !}(2 n-3) ! !, n \geq 2 \quad \text { and } \Delta^{1} f(k)=\frac{(2 k) ! !}{(2 k+1) ! !} .
$$

Hence, $\Delta^{n} f(0)=(-1)^{n-1} \frac{(2 n-3) ! !}{(2 n-1) ! !}=\frac{(-1)^{n-1}}{2 n-1}$,i.e.

$$
\sum_{k=0}^{s}(-1)^{k}\left(\begin{array}{l}
s \\
k
\end{array}\right) \frac{(2 k) ! !}{(2 k-1) ! !}=-\frac{1}{2 s-1}
$$

Then

$$
\begin{aligned}
A & =e^{-1}\left(-\sum_{s=0}^{\infty} \frac{(-1)^{s}}{(2 s-1) s !}+\sum_{s=0}^{\infty} \frac{(-1)^{s}}{(2 s-1) s !}-i \sum_{s=0}^{\infty} \frac{1}{s !(2 s-1)}\right) \\
& =-i e^{-1} \sum_{s=0}^{\infty} \frac{1}{s !(2 s-1)}=i \sum_{s=0}^{\infty}(-1)^{s} \frac{2^{s}}{(2 s-1) ! !}
\end{aligned}
$$

\section{References}

[1] D. S. Mitrinović, J. D. Kečkić, Metodi Izračunavanja Konačnih Zbirova, Beograd, 1990.

[2] M. R. Spiegel, Theory and Problems of Complex Variables, Singapore, 1988.

[3] K. Trenčevski, Ž. Tomovski, A solution of one old problem, to appear in Mathematical Bulletin, Skopje.

Institute of Mathematics, St. Cyril and Methodius University, P. O. Box 162, 1000 Skopje, Macedonia.

E-mail: tomovski@iunona.pmf.ukim.edu.mk

E-mail: kostatre@iunona.pmf.ukim.edu.mk 